\title{
Management of Phenytoin Induced Gingival Overgrowth: A Case Report
}

\author{
Batmaraj Rawisandran ${ }^{1}$, Radhika Arjunkumar ${ }^{2}$ \\ ${ }^{1}$ BDS CRRI Department of Periodontics, Saveetha Dental College, India \\ ${ }^{2}$ Senior Lecturer, Department of Periodontics, Saveetha Dental College, India
}

\begin{abstract}
Drug induced gingival enlargement is a gingival condition which is one of the common side effect with consumption of the drug phenytoin sodium. It is of vital importance to make the patients aware of the possibility of gingival overgrowth as soon as they are prescribed these drugs and inform them about the possible treatment options available. The main aim of the treatment is to minimize the growth and also the inflammatory component which can be achieved by surgical methods after assessment of the patient's medical condition. The choice of treatment lies in the hands of the dentist and it should be decided after careful assessment of all the factors associated with the disease. This case report documents a case of gingival enlargement associated with periodontitis in a patient under pheytoin sodium for the past 15 years, along with brief review of literature discussing the etiopathogenesis, and provides a brief protocol for its clinical management.
\end{abstract}

Keywords: Case report, drug induced gingival overgrowth, flap surgery, gingivectomy, phenytoin sodium

\section{Introduction}

Phenytoin sodium is an anti-epileptic drug which was first introduced around early 1938 [1].It is used to treat partial seizures and generalized tonic-clonic seizures. Although phenytoin is able to control seizures in patients it has its own side effects. The most common and widely known complication of phenytoin is gingival enlargement. Many terms have been used to describe gingival overgrowth or enlargement, some being, gingival hyperplasia which means " abnormal increase in the number of normal cells in a normal arrangement in an organ or tissue" and the expression gingival hypertrophy which means " enlargement or overgrowth of an organ or part due to an increase in size of its constituent cells" although gingival overgrowth is the general term that better describes this condition.

Kimball in 1939 [2] first reported the association of gingival enlargement with chronic use of phenytoin. About roughly in the same year and in 1959, Faurbye [3], Strean \& Leoni [4] reported that gingival enlargement is an effect of the alkalinity of phenytoin. Even though there have been several research and studies conducted regarding phenytoin-induced enlargement, the key pathogenesis behind the hyperplastic change in the gingival still remain clueless. Literature shows that there is a huge possibility which leads to the gingival changes such as multiple anti-epileptic therapies, plaque deposition, host genetic factors, and reduced serum folate levels [5]. The general agreement would be that, gingival hyperplasia is seen in patients under pheytoin is mainly the consequence of the increase in amount of connective tissue rather than a marked epithelial hyperplasia [6-7]. Literatures also suggest that major change in phenytoin gingival hyperplasia is due to the increase number of collagen but it still remains unclear if its caused by the increased number of fibroblast or elevated cellular activity[8-11].

In India, prevalence of gingival enlargement is roughly in between 15 to $50 \%$ [12]. Children aged between 8 to 13 years old have been reported to develop gingival enlargement in the first 6 months of intake of phenytoin [12]. It has been reported that upon withdrawal of phenytoin, gingival enlargement resolves spontaneously in approximately 1-6 months [13-14].

\section{Case Report}

A 35 year old female patient reported to our department complaining of progressive swelling and pain in the lower right front tooth region and pain in the lower left back tooth region for the past 1 month. History reveals that the patient has been taking the drug ( Eptoin) phenytoin sodium for seizure control approximately for the past 15 years. Intra oral examination reveals moderate overgrowth of the inter dental papilla in relation to 44 and 43 and the attached gingiva of 43 which is firm, and dense in consistency.(Fig. 1). Full mouth periodontal charting which includes assessment of probing depth and clinical attachment level was done and shows deep pockets which is more than $6 \mathrm{~mm}$ depth in relation to 41, 43, 31, 32, 36, 37 and 38 (Fig. 2-3). Patient had a poor oral hygiene status. Radiograph reveals angular bone loss in relation to 36, 37 and 37, 38 ,horizontal bone loss in relation to 31, 41 and 31, 32 (Fig. 4-5). 
Patient was then referred back to her own neurophysician for opinion, if any form of dental procedures could be conducted and if the patient needed any form of change in medication to control the gingival enlargement. Since the dosage was already tapered down to half ( 2 yrs ago) and phenytoin was prescribed along with sodium valporate, the patient reported back with no changes in the medication. The change of drug was well compensated showing no episode of recurrent seizure activity. Hence, she was considered fit to undergo ant dental procedure under local anesthesia.

\subsection{Treatment plan}

\section{Dental Management}

A complete treatment plan was made for the patient. Treatment was planned in 2 phases, where in the $1^{\text {st }}$ phase, a complete supra and sub gingival scaling along with root planing where deep pockets are present was scheduled. In phase 2 which approximately after 2 weeks, curettage in the region 41, 43, 31, 32, 36, 37 and 38 was planned followed by gingivectomy in relation to 44, 43 region. Flap surgery was scheduled in the region 36 , $37,31,41$. Each treatment was inclusive of follow up and recall.

\subsection{Treatment summary}

Initially a complete ultra sonic scaling and root planing is done. Patient was recalled after 2 weeks. Decreases in pocket depths were not significant in the region of 44, 43, 41, 31, 36, 37 and 38. Patient was then scheduled for curettage.

Curettage was completed in relation to 44, 43, 41, 31, 36, 37 and 38. Pocket depth was measured again after 2 weeks and there was slight decrease but futher invasive management was required in relation to 44, 43, 41, 31, 36, 37 and 38. Patient was then scheduled for gingivectomy in 43, 44 region and for flap surgery in relation to $41,31,36,37$ and 38 . Complete hematological investigation was carried out and the results were found to be normal. Full thickness mucoperiosteal flap was elevated in 36, 37, 38 region (Fig. 6). Complete debridement was done. Horizontal bone loss and angular defects were seen in 363738 region. Because the defects were shallow and non containing no attempt to regenerate the bone was made. Elevated mucosa was approximated by a sling suture. A papilla preservation flap was elevated in relation to 31, 4142 region (Fig. 7). Complete debridement was done. . Elevated mucosa was approximated by a simple interrupted suture.

Gingivectomy was done in the region 43, 44 (Fig. 8) where the gingival enlargement was present. \#15 Bard-Parker blade was placed on the marginal gingiva in the coronal direction to a point between the base of the pocket and the alveolar crest and the gingival overgrowth was excised. Soft tissue pocket was also eliminated. Tissue biopsy was done, specimen tissue was sent for histopathological examination. Periodontal dressing was given at the region $31,32,41,43,44$ to cover the wound. Analgesics and antibiotics were prescribed. Patient was asked to report after 2 week to check for healing status. Oral hygiene instructions were given.

Patient was recalled after 2 week and one month after surgery to observe post operative results. Healing was satisfactory (Fig. 9-11). Patient is now placed under a regular maintenance protocol to check for any recurrence of overgrowth. There was no recurrence of gingival overgrowth until her last post operative visit.

\section{Histopathological findings}

Histopathological examination of the excised gingival tissue was done using eosin and hematoxylin stains. Section shows parakeratinised stratified squamous epithelium of variable thickness with features of hyperplasia and changes secondary to the underlying inflammation. There is evidence of intense chronic inflammatory cell infiltration in the underlying connective tissue stroma (Fig 12).

\section{Discussion}

Gingival enlargement is a common side effect among patients under phenytoin therapy. It usually develops in susceptible individuals at usually within 1 to 3 months of starting the medication [15]. It is a soft tissue growth which initially starts as a painless, bead-like, diffuse swelling at the region of the interdental papilla, which later on enlarges and combines to form a nodular form of a swelling. As the swelling progresses to the marginal gingiva it form a massive tissue fold which sufficiently covers a huge portion of the tooth structure [16]. It does not cause any form of tenderness unless it is secondarily infected. Secondary inflammatory changes not only add to the size and tenderness of the lesion caused by the drug, but also produce a red or bluish red discoloration, obliterating the lobulated surface demarcations and increasing the tendency to bleed. It has been reported that gingival overgrowth in individuals prescribed with phenytoin can be minimized with appropriate personal oral hygiene and professional maintenance [17-18]. The presence of the enlargement makes the plaque control and maintenance of the area difficult, which results in secondary inflammatory response which complicates the overgrowth. A 3 month interval for periodontal maintenance is necessary in patients who are under pheyntoin [14]. Gingival enlargement does not occur in all patients under phenytoin, if it occurs, it can be classified as stated in table 1 [20]. 


\begin{tabular}{|l|l|}
\hline TYPE 1 & $\begin{array}{l}\text { Non- inflammatory hyperplasia. Substitution of phenytoin with another anti- epileptic drug is the only method of eliminating } \\
\text { this hyperplasia. Subsequent to substitution, the enlargement disappears after a few months. }\end{array}$ \\
\hline TYPE 2 & $\begin{array}{l}\text { Chronic inflammatory enlargement not related to phenytoin use. This enlargement is caused entirely by local irritants, and } \\
\text { resembles inflammatory enlargement in patients not receiving phenytoin } .\end{array}$ \\
\hline TYPE 3 & Combined enlargement. This is a combination of hyperplasia caused by phenytoin and inflammation by local irritation \\
\hline
\end{tabular}
table 1

Drug substitution would be one of the most important steps in treating and preventing recurrence of gingival enlargement [6, 19-20]. Newer drugs such as lomatrigine, gabapentin, sulthiame, and topiramate can be opted as a drug substitute. Proper personal oral hygiene and professional maintenance said to have a major role in minimizing gingival overgrowth among patients who are under phenytoin [14, 17-18]. Other predisposing factors such as bacterial plaque can also influence the treatment out come. It is of at most importance that proper oral prophylaxis is done prior to any surgical intervention. However, the remaining residual gingival overgrowth inhibits plaque control and may also present as aesthetic and functional problem [21]. In such cases periodontal surgery would be the treatment option: either gingivectomy or periodontal flap. Type of surgery is determined by evaluating the extent of the area to be treated, any osseous defects present along with the gingival enlargement, position of the bases of the pocket in relation to the existing mucogingival junction. According to Camargo PM et al. [22] the need for flap surgery or gingivectomy can be determined based of certain rules. Gingivectomy is indicated in cases where, small areas $(\leq 6$ teeth) presenting with drug-induced gingival enlargement (there is no evidence of attachment loss and therefore no anticipated need to perform osseous surgery). But ideally, there should be at least $3 \mathrm{~mm}$ of keratinized tissue in the apico-coronal direction remain after the surgical procedure. Whereas periodontal flap is indicated in cases involving larger areas of gingival enlargement ( $>6$ teeth), or areas where attachment loss combined with osseous defects is present.

Laser treatment can also be a mode of treatment in esthetic corrections in cases where gingivectomy is indicated. Soft tissue laser provides minimal pain and discomfort during and after the procedure [6]. It also promotes faster healing in such cases. Laser would be another good inclusion in the treatment protocol when it comes to treating patients with phenytoin induced gingival enlargement.

A multidisciplinary treatment and more rational anti-epileptic therapy would by whole treat this problem more effectively.

Recurrence of drug-induced gingival enlargement is very common in surgically treated cases [23]. It can occur as early 3-6 month but usually surgical results can be maintained for atleast 12 months [22]. The degree of recurrence can be reduced by implementing meticulous home care, chlorhexidine gluconate rinses, and professional cleaning.

VI. Figures

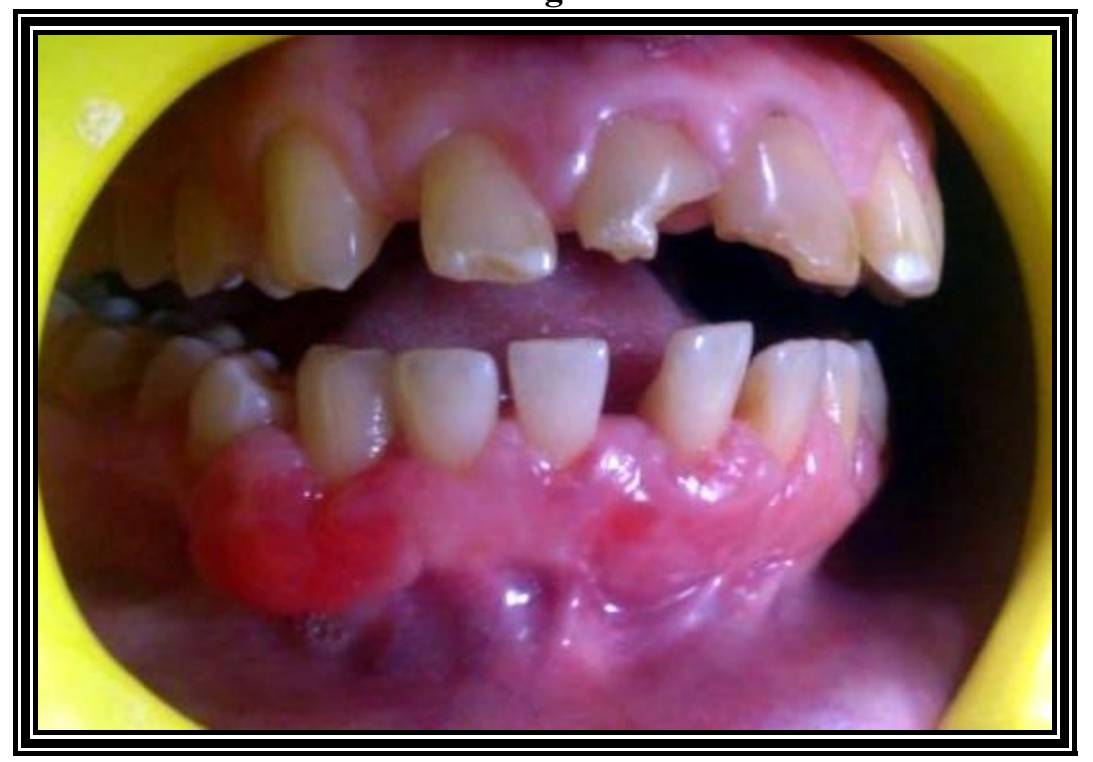

figure 1- preoperative photograph 


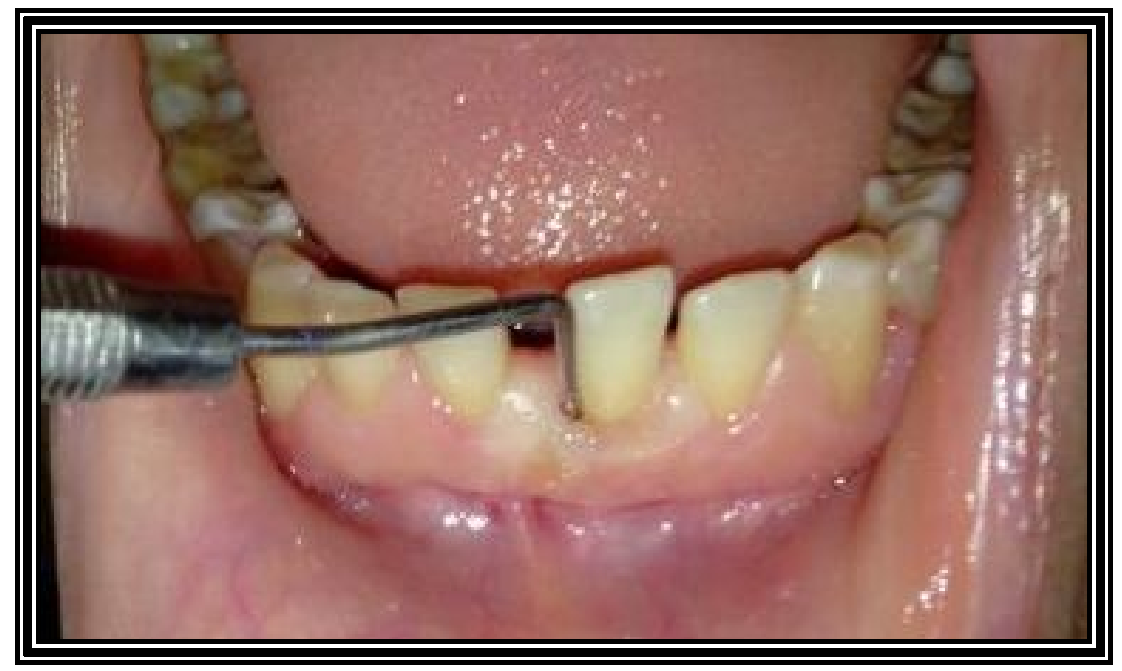

figure 2- probing depth in relation to 31

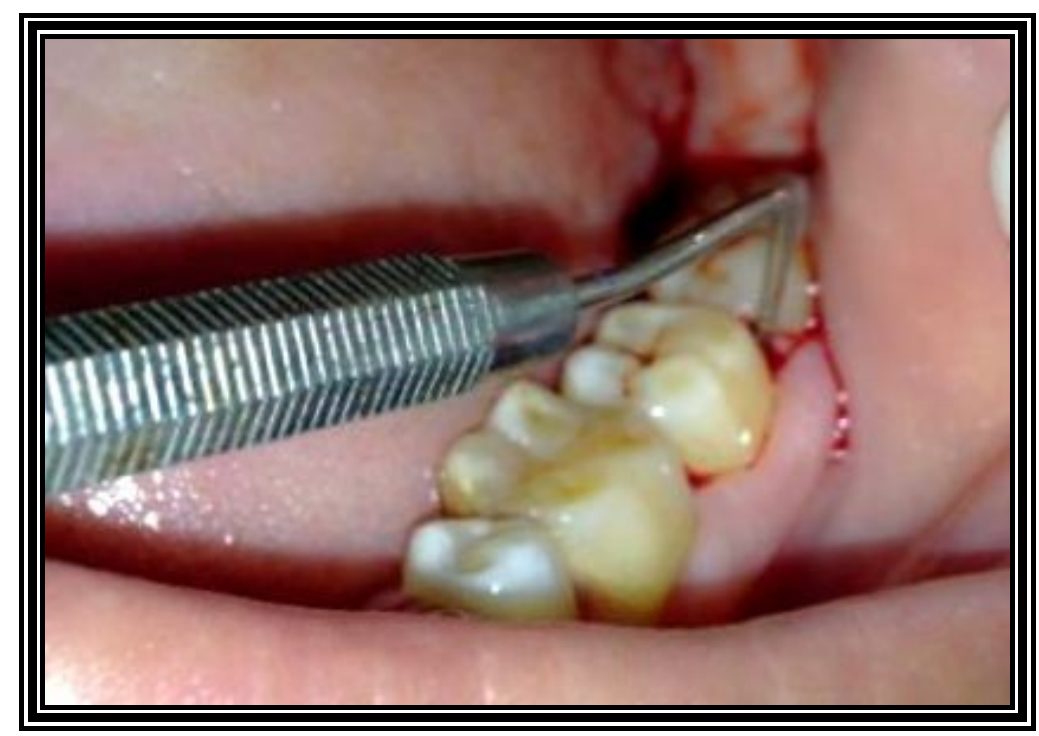

figure 3- Probing depth in relation to 38

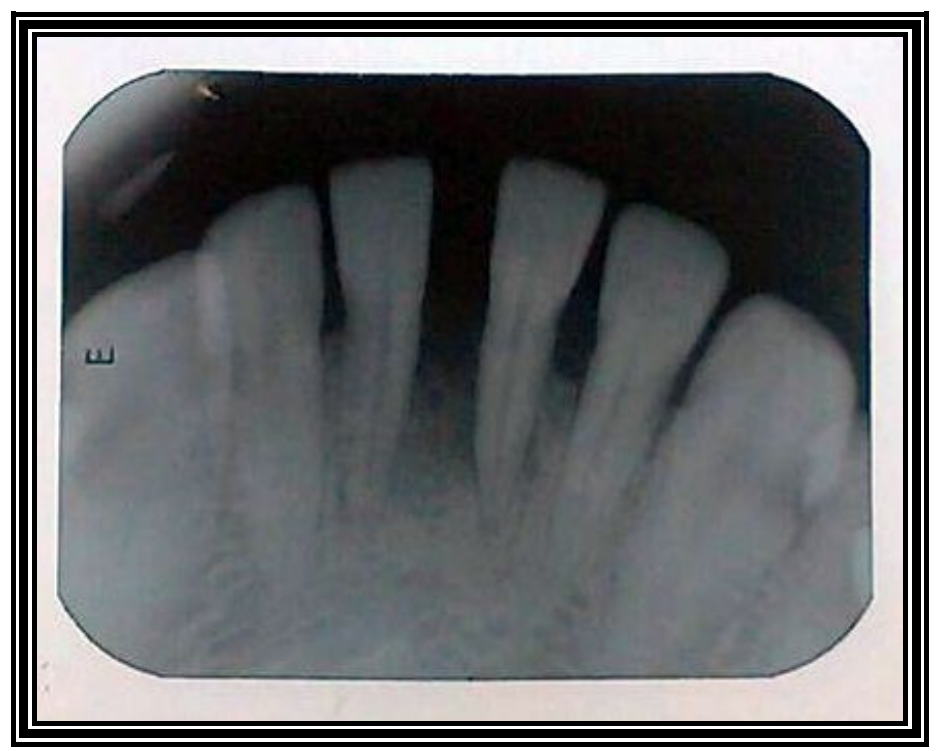

figure 4- Intraoral radiograph shows horizontal bone lost in relation to 31,41 and 42 . 


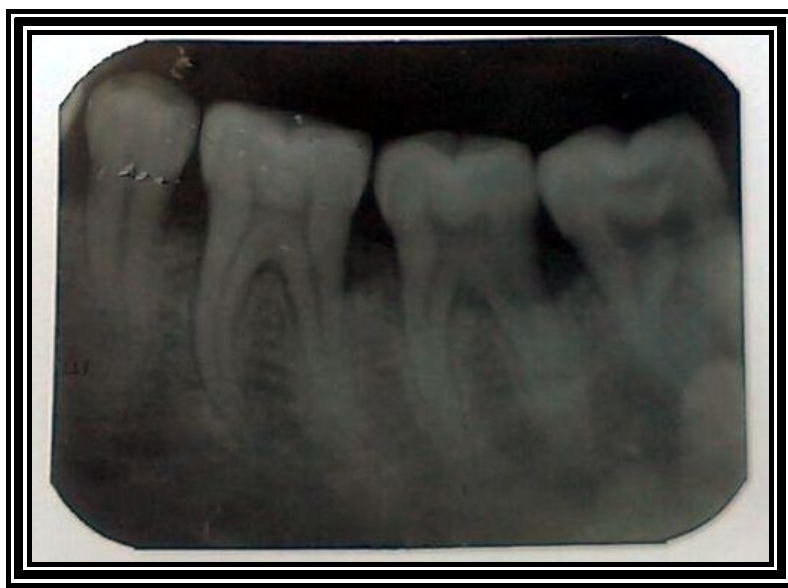

figure 5- Intraoral radiograph shows horizontal bone loss in 36, 37

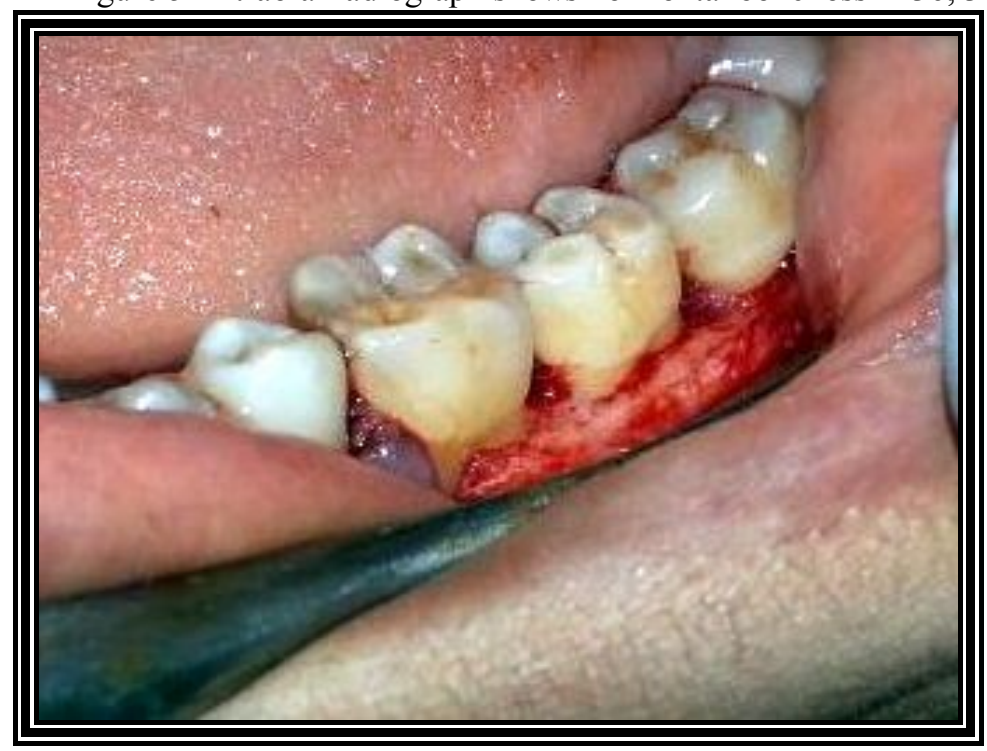

figure 6- Flap elevation in relation to $35,36,37,38$.

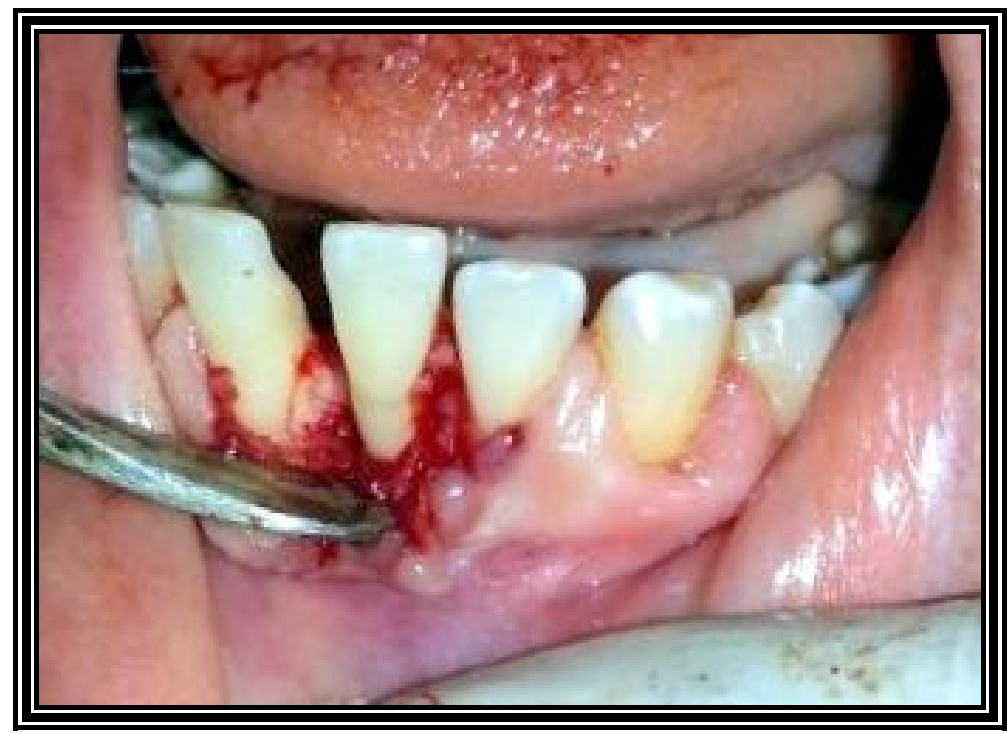

figure 7- Flap elevation in relation to $31,41,4$ 


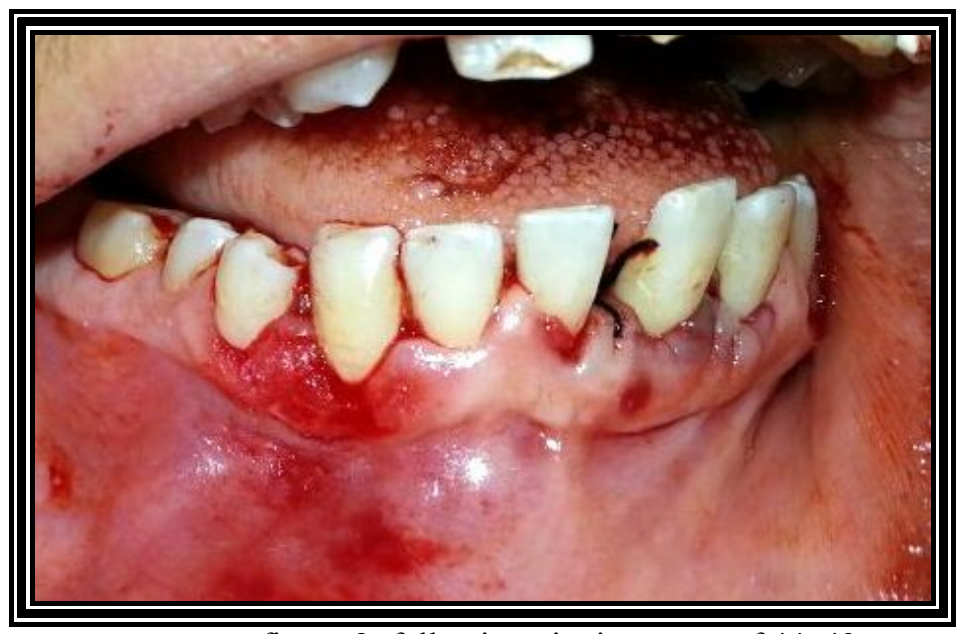

figure 8 - following gingivectomy of 44,43

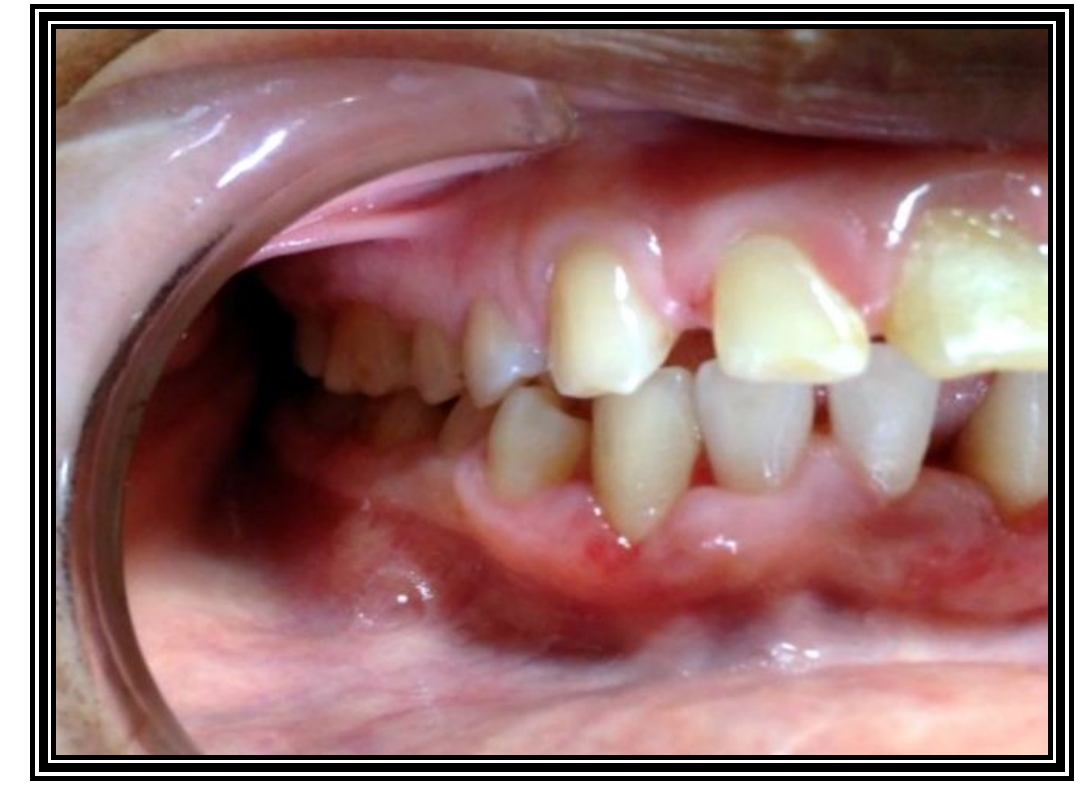

figure 9- post operative status after 2 weeks

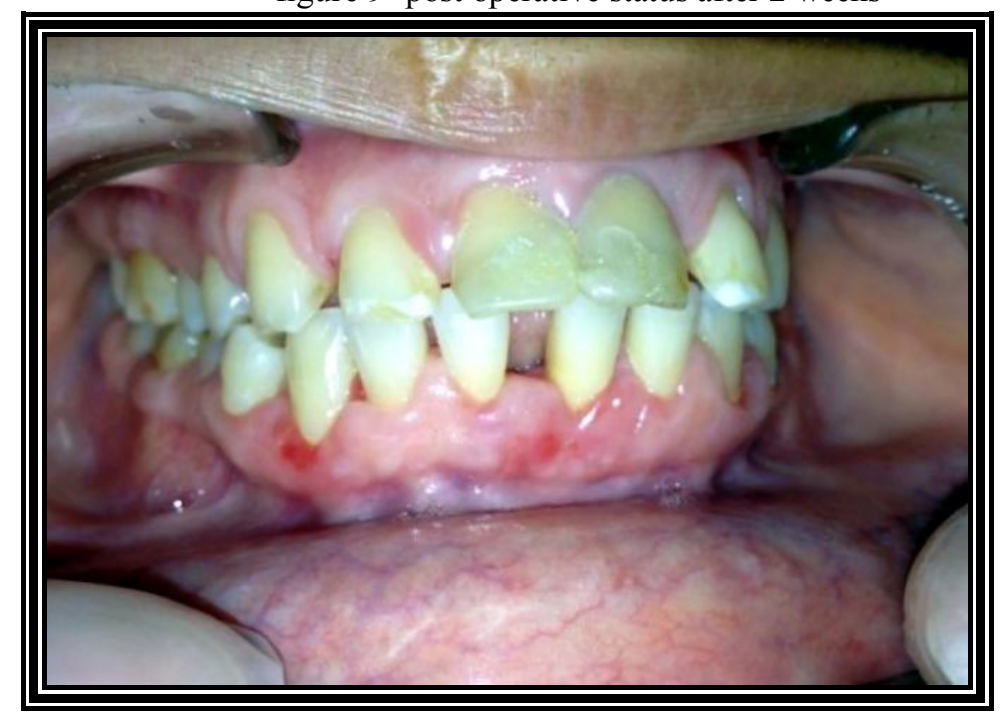

figure 10- post operative status after 2 week 


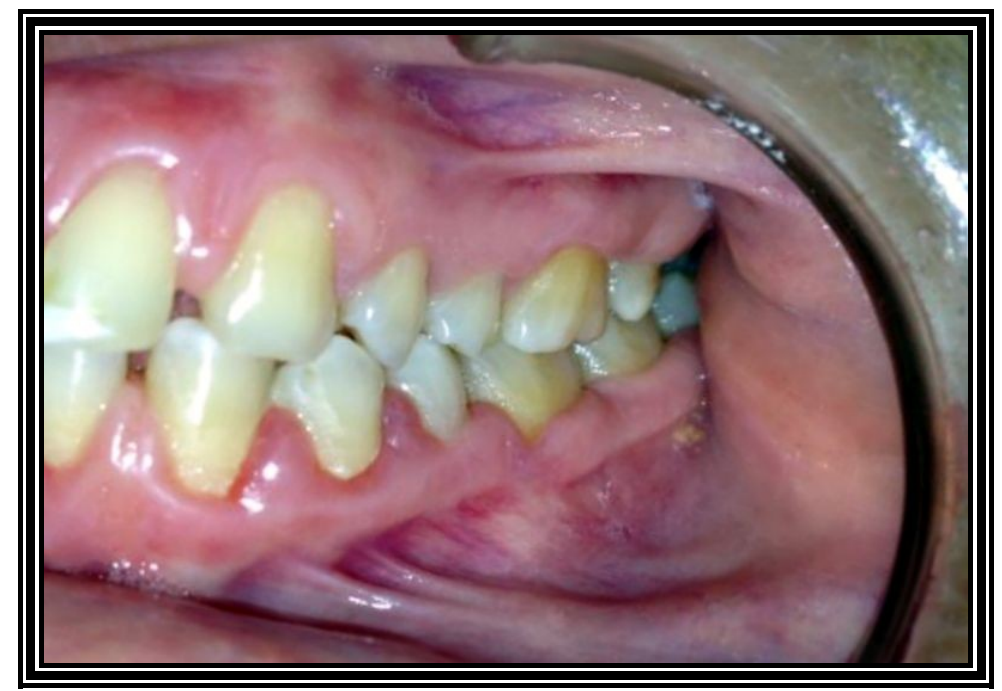

figure 11- post operative status after 2 weeks

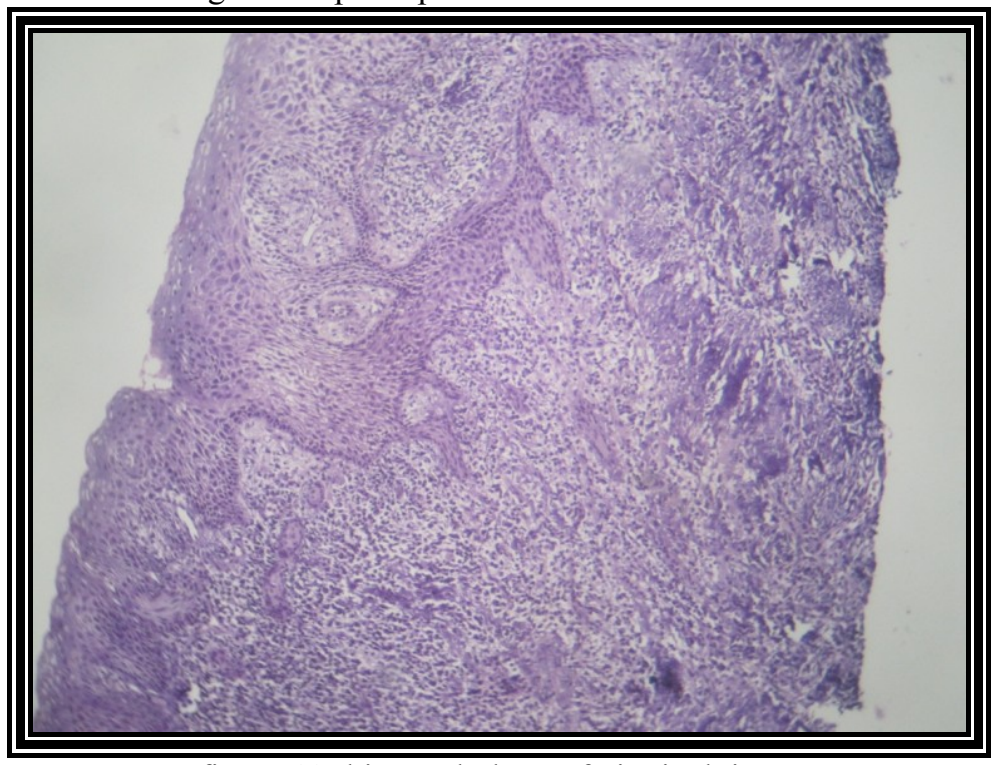

figure 12- histopathology of gingival tissue

\section{Conclusion}

It has been a known fact that periodontal tissue is very susceptible to adverse effects caused by a range of systemic drug therapy. This stresses the importance of regular medical and oral checkups which includes periodontal screening and oral health maintenance. Phenytoin-induced gingival enlargement is a problem which can directly or indirectly caused by the effect of the drug phenytoin. For an effective control of this problem, proper treatment protocol would be necessary. Treating the excessive tissue enlargement alone without considering other factors such as the drug and local factors such as plaque and calculus will not completely benefit the patient. It is very crucial to include drug substitution and control of local inflammatory factors as a initial part of treatment protocol. However, when this sequence of treatment fails to resolve the problem, surgical intervention can provide favorable results.

\section{References}

[1]. Merritt H, Putman T, Sodium diphenyl hydantoinate in the treatment of convulsive disorders. JAMA, 111, 1938, 1068-1103.

[2]. Kimball OP, The treatment of epilepsy with sodium diphenyl hydantoinate, J Am Med. Assoc., 112, $1939,1244-1245$.

[3]. Faurbye A, Rehandling of epilepsy med diphenylhydantoin, Ugeskr Laeg, 101, 1939, 1350-1354.

[4]. Strean LR, Leoni E, Dilantin gingival hyperplasia. Newer concepts related to etiology and treatment, NY St Dent J, 25, 1959, 339347

[5]. Vogel R.I, Gingival hyperplasia and folic acid deficiency from anticonvulsive drug therapy: A theoretical relationship, $J$ Theor Biol, 67, 1977, 269-278

[6]. Marshall RI, Bartold PM, A clinical review of drug-induced gingival overgrowth, Aust Dent J, 44, 1999, $219-232$

[7]. Keith, D.A., Paz, M.A. and Gallop, P.M, The effect of diphenylhydantoin on fibroblasts in vitro, J.Dent. Res. 56 (10), 1977,1279 1283.

[8]. Angelopoulos, A.P, Diphenylhydantoin gingival hyperplasia: A clinicopathologic review, J.Can. Dent. Assoc, 41, 1975,103 - 106. 
[9]. Bienkowski, R.S, Intracellular degradation of newly synthesized secretory protein, Biochem. J, 83, 1983, 214

[10]. Keith, D.A., Paz, M.A. and Gallop, P.M. (1977), The effect of diphenylhydantoin on fibroblasts in vitro. J.Dent. Res. 56 (10), 1977, $1279-1283$.

[11]. Kerr, D.A, Stomatitis and gingivitis in the adolescent and the pre-adolescent, J.Am. Dent. Assoc. 44, 1952, $27-34$

[12]. Mejia LM, Lozada-Nur F, Drug induced gingival hyperplasia, E medicins. Medscope. com 2009; articles / 1076264

[13]. Sanders B, Weddell JA, Dodge NN, Managing patients who have seizure disorders, Dental and medical issues, J Am Dent Assoc, $126,1995,1641$

[14]. Hall EE, Prevention and treatment consideration in patients with drug induced gingival enlargement, Curr Opin Periodontol, 4, 1997, 59-63.

[15]. Meraw SJ, Sheridan PJ, Medically induced gingival hyperplasia, Mayo Clin Proc, 73, 1998, 1196-1199

[16]. Carranza FA, Newman MG, Gingival enlargement, Clinical Periodontology 8 (Pheiledelpia: W.B: Saunders Company 1996) 233249

[17]. Pihlstrom B, Prevention and treatment of dilantin associated gingival enlargement, Compend Contin Educ Dent 1990;11 (suppl 14), 506-510.

[18]. Hall WB, Dilantin hyperplasia: A preventable lesion? Compend Contin Educ Dent, 11(suppl 14) , $1990,502-505$.

[19]. Brunsvold M, Tomasovic J, Reumping D, The measured effect of phenytoin withdrawal on gingival hyperplasia in children, $J C l i n$ Periodontol, 19, 1992, 845-849.

[20]. Dahllof G,Axio E, Modeer T, Regression of pheyntoin-induced gingival overgrowth after withdrawal of medication, Swed Dent J, $15,1991,139-143$.

[21]. Jones JE, Weddell JA, McKown CG, Incidence and indications for surgical management of phenytoin-induced gin-gival overgrowth in a cerebral palsy population, J Oral Maxillofac Surg, 46, 1998, 385-90.

[22]. Camargo PM, Melnick PR, Pirih FQ, Lagos R, Takei HH, Treatment of drug-induced gingival enlargementant: aesthetic and functional considerations, Periodontol 2000, 27, 2001, 131-138

[23]. Rees TD, Levine RA, Systemic drugs as a risk factor for periodontal disease initiation and progression. Compendium. 1995, 16(1), 20,22,26 passim; quiz 42 . 